\title{
Bilinear amplitude approximation for piecewise-linear oscillators
}

\author{
Chulwoo Jung, Kiran D’Souza, and Bogdan I. Epureanu* \\ Department of Mechanical Engineering \\ University of Michigan, Ann Arbor, MI 48109-2125
}

\section{Introduction}

Currently, the interest in monitoring the vibration of dynamical systems has been increasing. For example, large and complex air and space structures include vibration monitoring systems to forecast sudden failures. Such vibration monitoring is used to diagnose structural health by analyzing vibration characteristics (such as frequency and amplitude). Among the vibration characteristics used, the vibration amplitude is especially important because it can directly affect the life of the system.

Finite element (FE) models are often used to analyze vibration characteristics. For low dimensional systems, full FE models can be used. For high dimensional systems, the computational cost of analyzing full FE models can often be prohibitive. To circumvent this difficulty, many methods for creating reduced-order models (ROMs) have been developed for various systems [1-14], with the majority being focused on linear systems.

ROMs for linear systems can be efficiently constructed by using approaches based on linear transformations $[15,16]$ such as component mode synthesis (CMS) [17]. However, constructing ROMs for systems with piecewise-linear nonlinearity (caused for example by intermittent contact) require careful treatment. Accurate ROMs can be constructed for such systems using linear transformations [18-20], or using nonlinear normal modes [21-23]. Recently, Saito et al. [24] developed a reduced-order modeling method based on bilinear modes (BLMs) for dynamical systems with piecewise-linear nonlinearity. They observed that the space spanned by the most dominant proper orthogonal modes (POMs) of a system is also spanned by a set of linear normal modes for the system with special boundary conditions at the surface where the intermittent contact takes place. The special modes were referred to as BLMs. Hence, the most dominant POMs are well approximated by linear combinations of BLMs. Thus, ROMs based on BLMs are accurate and have a low dimension. Nonetheless, predicting the vibration amplitude requires the calculation of the nonlinear forced response of the ROMs. Namely, the nonlinear forced responses have to be obtained by direct numerical calculation (e.g., by using a variable step Runge-Kutta method), which incurs a large computational cost despite the fact that the ROMs are low dimensional. For example, mistuned bladed disks with cracks have piecewise-linear nonlinearity due to the intermittent contact at the crack surfaces. Therefore, to obtain the amplitude of vibration at the resonant frequencies, nonlinear forced responses need to be calculated using more efficient numerical methods (e.g., hybrid frequency/time domain methods) [25-28].

In this paper, a novel technique to approximate the vibration amplitude at the resonant frequencies of dynamical systems with piecewise-linear nonlinearity is proposed. Here, it is assumed that the forcing applied to the system is harmonic and the response of the system is periodic. Thus, quasi-periodic or chaotic dynamics are not considered. The proposed technique is referred to as bilinear amplitude approximation (BAA). BAA constructs approximations for the periodic steady-state response of the system at resonant frequencies. For example, consider that a structure has a crack which opens and closes during each vibration cycle. BAA uses linear modes (similar to BLMs) from two different systems: one with an open crack and the other where there is sliding at the crack surfaces. By doing so, BAA does not require the numerical integration of nonlinear ROMs to calculate the vibration amplitude at resonant frequencies. Consequently, large savings in computational costs are obtained.

\footnotetext{
${ }^{*}$ Corresponding author: B. I. Epureanu, epureanu@umich.edu
} 
In the following, BAA is introduced. Then, results comparing exact numerical solutions and results obtained using BAA for a full blisk with a crack are presented. Finally, conclusions are presented.

\section{Methodology}

In this section, BAA is introduced. Consider an elastic structure which undergoes intermittent contact (leading to piecewise-linear nonlinearity). During each vibration cycle, the structure has three different states: (1) fully open (i.e., no contact), (2) fully sliding (i.e., complete contact), and (3) partially open (i.e., partial contact between the contacting surfaces). The goal of BAA is to find the steady-state amplitude of vibration of the system when excited by harmonic forcing under the following assumptions: (a) state (3) (partially open) lasts a much shorter time interval than states (1) and (2), (b) the motion of the structure is periodic, (c) during each vibration cycle there is only one time interval when the system is in state (1) and only one time interval when the system is in state (2); thus, one entire vibration cycle is approximated by states $(1)$ and (2), and (d) the motion in state (1) is dominated by one mode of the structure with fully open contact, and the motion in state (2) is dominated by one mode of the structure with fully sliding at the contact surfaces. Assumption (a) is in fact exactly satisfied when the gap at the contacting surfaces is zero for the structure with zero internal stresses [24,27].

The motion of one steady-state vibration cycle for one of the degrees-of-freedom of the system is schematically shown in Fig. 1. The total period $T$ of the periodic response is broken into the time $T_{o}$ that the system spends in state $(1)$, and the time $T_{s}$ that the system spends in state (2). The fraction of the entire period $T$ that the system is in state $(2)$ is $f=T_{s} / T$.

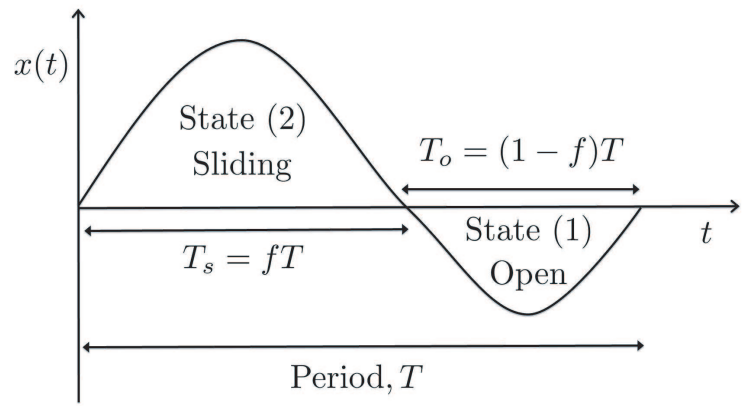

Figure 1: One steady-state vibration cycle

Consider a multi-degree-of-freedom system. The exact equations which govern the motion in state (1) and in state (2) can be expressed as

$$
\begin{aligned}
\mathbf{M}_{o} \ddot{\mathbf{x}}_{o}(t)+\mathbf{C}_{o} \dot{\mathbf{x}}_{o}(t)+\mathbf{K}_{o} \mathbf{x}_{o}(t) & =\mathbf{F}(t), \\
\mathbf{M}_{s} \ddot{\mathbf{x}}_{s}(t)+\mathbf{C}_{s} \dot{\mathbf{x}}_{s}(t)+\mathbf{K}_{s} \mathbf{x}_{s}(t) & =\mathbf{F}(t),
\end{aligned}
$$

where the subscript $o$ refers to state (1), the subscript $s$ refers to state (2), $\mathbf{M}_{o}$ and $\mathbf{M}_{s}$ are the mass matrices, $\mathbf{C}_{o}$ and $\mathbf{C}_{s}$ are the damping matrices, $\mathbf{K}_{o}$ and $\mathbf{K}_{s}$ are the stiffness matrices, and $\mathbf{F}$ is the external force. The mode shapes for the structure in states (1) and (2) are $\boldsymbol{\Phi}_{o}$ and $\boldsymbol{\Phi}_{s}$. A physical to modal transformation leads to $\mathbf{x}_{o}=\boldsymbol{\Phi}_{o} \mathbf{q}_{o}$ and $\mathbf{x}_{s}=\boldsymbol{\Phi}_{s} \mathbf{q}_{s}$, where $\mathbf{q}_{o}$ and $\mathbf{q}_{s}$ are modal coordinates. Assumption (d) implies that the motion in state (1) can be approximated well by a single dominant mode $\boldsymbol{\Phi}_{o}^{*}$, and the motion of the structure in state (2) can be approximated by a single dominant mode $\boldsymbol{\Phi}_{s}^{*}$. Thus, $\mathbf{x}_{o} \cong \boldsymbol{\Phi}_{o}^{*} q_{o}$ and $\mathbf{x}_{s} \cong \boldsymbol{\Phi}_{s}^{*} q_{s}$. Also, Eq. (1) can be projected along the vectors $\boldsymbol{\Phi}_{o}^{*}$ and $\boldsymbol{\Phi}_{s}^{*}$ to obtain

$$
\begin{aligned}
& \boldsymbol{\Phi}_{o}^{* \mathrm{~T}} \mathbf{M}_{o} \boldsymbol{\Phi}_{o}^{*} \ddot{q}_{o}+\boldsymbol{\Phi}_{o}^{* \mathrm{~T}} \mathbf{C}_{o} \boldsymbol{\Phi}_{o}^{*} \dot{q}_{o}+\boldsymbol{\Phi}_{o}^{* \mathrm{~T}} \mathbf{K}_{o} \boldsymbol{\Phi}_{o}^{*} q_{o}=\boldsymbol{\Phi}_{o}^{* \mathrm{~T}} \mathbf{F}(t), \\
& \boldsymbol{\Phi}_{s}^{* \mathrm{~T}} \mathbf{M}_{s} \boldsymbol{\Phi}_{s}^{*} \ddot{q}_{s}+\boldsymbol{\Phi}_{s}^{* \mathrm{~T}} \mathbf{C}_{s} \boldsymbol{\Phi}_{s}^{*} \dot{q}_{s}+\boldsymbol{\Phi}_{s}^{* \mathrm{~T}} \mathbf{K}_{s} \boldsymbol{\Phi}_{s}^{*} q_{s}=\boldsymbol{\Phi}_{s}^{* \mathrm{~T}} \mathbf{F}(t),
\end{aligned}
$$

which leads to

$$
\begin{aligned}
& \ddot{q}_{o}+2 \zeta_{o} \omega_{o} \dot{q}_{o}+\omega_{o}^{2} q_{o}=f_{o} \\
& \ddot{q}_{s}+2 \zeta_{s} \omega_{s} \dot{q}_{s}+\omega_{s}^{2} q_{s}=f_{s}
\end{aligned}
$$




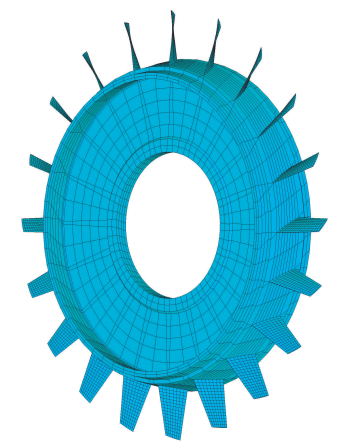

Figure 2: Bladed disk model

where $\zeta_{o}$ and $\zeta_{s}$ are the viscous damping ratios, $\omega_{o}$ and $\omega_{s}$ are the undamped natural frequencies associated with $\boldsymbol{\Phi}_{o}^{*}$ and $\boldsymbol{\Phi}_{s}^{*}$, and $f_{o}$ and $f_{s}$ represent modal forcing. Using Eq. (3), the modal coordinates corresponding to the linear modes $\boldsymbol{\Phi}_{o}^{*}$ and $\boldsymbol{\Phi}_{s}^{*}$ can be expressed as

$$
\begin{aligned}
& q_{o}(t)=\exp ^{-\zeta_{o} \omega_{o} t}\left(o_{1} \cos \left(\omega_{o d} t\right)+o_{2} \sin \left(\omega_{o d} t\right)\right)+\frac{\left(f_{o} / \omega_{o}^{2}\right) \cos \left(\omega t-\theta_{o}+\alpha\right)}{\sqrt{\left(1-\left(\omega / \omega_{o}\right)^{2}\right)^{2}+\left(2 \zeta_{o} \omega / \omega_{o}\right)^{2}}}, \\
& q_{s}(t)=\exp ^{-\zeta_{s} \omega_{s} t}\left(s_{1} \cos \left(\omega_{s d} t\right)+s_{2} \sin \left(\omega_{s d} t\right)\right)+\frac{\left(f_{s} / \omega_{s}^{2}\right) \cos \left(\omega t-\theta_{s}+\alpha\right)}{\sqrt{\left(1-\left(\omega / \omega_{s}\right)^{2}\right)^{2}+\left(2 \zeta_{s} \omega / \omega_{s}\right)^{2}}},
\end{aligned}
$$

where $o_{1}, o_{2}, s_{1}$, and $s_{2}$ are scalar coefficients, $\omega_{\text {od }}$ and $\omega_{s d}$ are the damped frequencies corresponding to the natural frequencies $\omega_{o}$ and $\omega_{s}, \theta_{o}=\arctan \left(\frac{2 \zeta_{o} \omega_{o} \omega}{\omega_{o}^{2}-\omega^{2}}\right), \theta_{s}=\arctan \left(\frac{2 \zeta_{s} \omega_{s} \omega}{\omega_{s}^{2}-\omega^{2}}\right)$, and $\alpha$ is an angle that reflects the phase difference between the steady-state response and the forcing.

There are six unknowns in Eq. (4): the phase angle $\alpha$, the time fraction variable $f$, and the four coefficients $o_{1}, o_{2}, s_{1}$, and $s_{2}$. Similar to the arguments used in bilinear frequency approximations (BFA) [29], $T_{o}$ and $T_{s}$ can be approximated by $T_{o} \cong \frac{2 \pi}{\omega_{o}}$ and $T_{s} \cong \frac{2 \pi}{\omega_{s}}$. Thus, the fraction $f$ can be approximated by

$$
f_{a}=\frac{T_{s}}{T_{o}+T_{s}} \cong \frac{\omega_{o}}{\omega_{o}+\omega_{s}}
$$

To calculate the remaining 5 unknowns, one can enforce transition conditions related to conservation of energy and linear momentum at the instances when the system switches from state (1) to state (2) or vice versa. This yields transition conditions which are used to solve for the unknown coefficients in Eq. (4). One obtains

$$
\begin{aligned}
E_{s}\left(T_{s}\right) & =E_{o}\left(T_{s}\right), \\
E_{o}\left(T_{o}+T_{s}\right) & =E_{s}(0), \\
\mathbf{L}_{s}\left(T_{s}\right) & =\mathbf{L}_{o}\left(T_{s}\right), \\
\mathbf{L}_{o}\left(T_{o}+T_{s}\right) & =\mathbf{L}_{s}(0),
\end{aligned}
$$

where $E_{o}$ and $E_{s}$ represent the energy of the system in state (1) and (2), and $\mathbf{L}_{o}$ and $\mathbf{L}_{s}$ represent the linear momentum in state (1) and (2).

The first two relations in Eq. (6) enforce that the total energy is conserved as the system transitions between state (1) and state (2). The first relation corresponds to the case where the system moves from the sliding state to the open state. The energy of the system in state (2) as it is about to transition to state (1) is comprised of the strain energy $S E_{s}$ and kinetic energy $K E_{s}$ given by

$$
\begin{aligned}
S E_{s}\left(T_{s}\right) & =\frac{1}{2} \mathbf{x}_{s}^{\mathrm{T}}\left(T_{s}\right) \mathbf{K}_{s} \mathbf{x}_{s}\left(T_{s}\right), \\
K E_{s}\left(T_{s}\right) & =\frac{1}{2} \dot{\mathbf{x}}_{s}^{\mathrm{T}}\left(T_{s}\right) \mathbf{M}_{s} \dot{\mathbf{x}}_{s}\left(T_{s}\right) .
\end{aligned}
$$




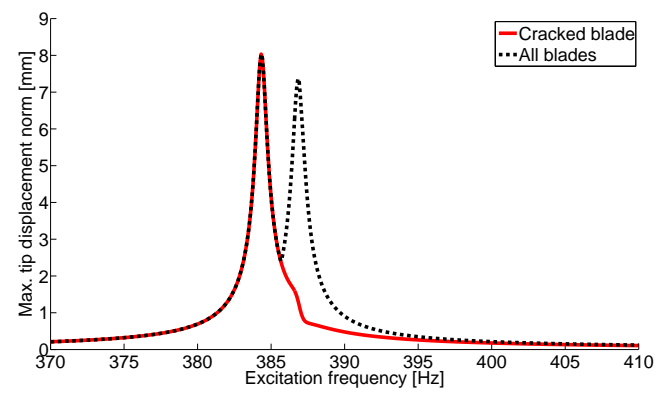

Figure 3: Nonlinear forced response of the $1^{\text {st }}$ mode family

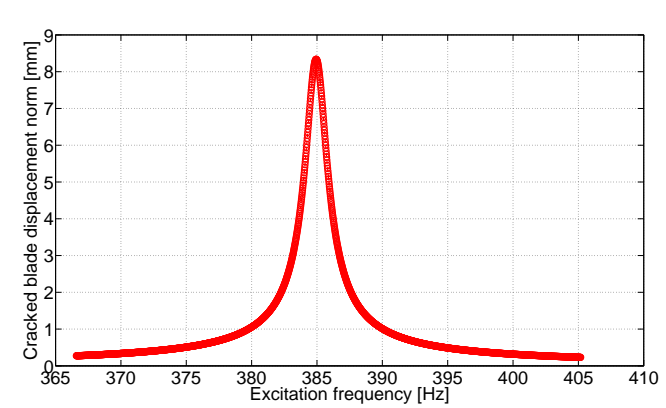

(a)

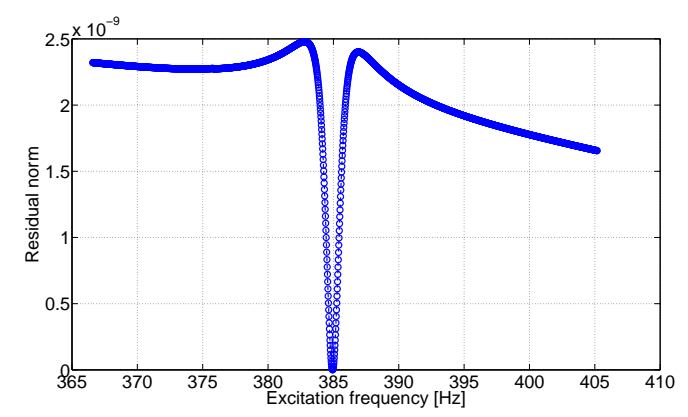

(b)

Figure 4: The (a) amplitude and (b) residual calculated using BAA for the $1^{\text {st }}$ mode family

Using the fact that the motion in state (2) is dominated by $\boldsymbol{\Phi}_{s}^{*}$, one obtains $\mathbf{x}_{s}(t) \cong \boldsymbol{\Phi}_{s}^{*} q_{s}(t)$ and $\dot{\mathbf{x}}_{s}(t) \cong \mathbf{\Phi}_{s}^{*} \dot{q}_{s}(t)$. Adding these relations to Eq. (7) one obtains

$$
\begin{aligned}
S E_{s}\left(T_{s}\right) & =\frac{1}{2} \omega_{s}^{2} q_{s}^{2}\left(T_{s}\right), \\
K E_{s}\left(T_{s}\right) & =\frac{1}{2} \dot{q}_{s}^{2}\left(T_{s}\right) .
\end{aligned}
$$

The energy in state (1) just after it transitions from state (2) is given by the strain energy $S E_{o}$, the kinetic energy $K E_{o}$

$$
\begin{aligned}
S E_{o}\left(T_{s}\right) & =\frac{1}{2} \omega_{o}^{2} q_{o}^{2}\left(T_{s}\right), \\
K E_{o}\left(T_{s}\right) & =\frac{1}{2} \dot{q}_{o}^{2}\left(T_{s}\right) .
\end{aligned}
$$

Also, the work done by the external forcing $W_{e x t}$ during the transition is given by

$$
W_{e x t}\left(T_{s}\right)=f_{o} q_{o}\left(T_{s}\right)-f_{s} q_{s}\left(T_{s}\right) .
$$

Similar expressions for the strain energy, kinetic energy and external work can be written for the second relation in Eq. (6). Namely, the conservation of energy for when the system moves from state (1) to state (2). In this case an additional coefficient $c_{e}$ corresponding to the loss of energy caused by the impact of the crack surfaces when the system moves from open to sliding must be defined. This coefficient is very close to zero and depends on the shapes of $\boldsymbol{\Phi}_{o}^{*}$ and $\boldsymbol{\Phi}_{s}^{*}$ in the vicinity of the crack.

The third and fourth relations in Eq. (6) enforce conservation of linear momentum for the system when the system transitions between state (1) and state (2) (which is assumed to occur over a negligible amount 


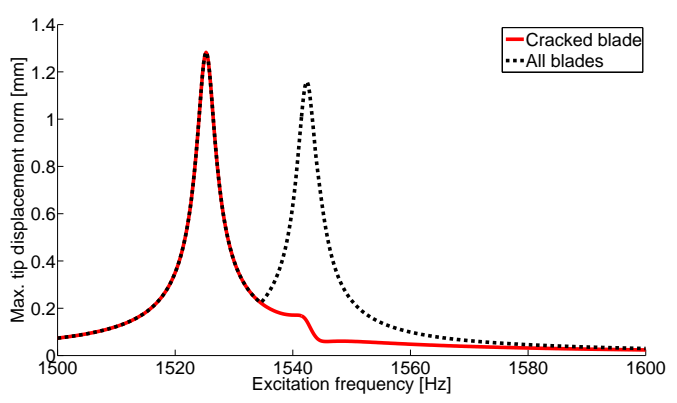

Figure 5: Nonlinear forced response of the $2^{\text {nd }}$ mode family

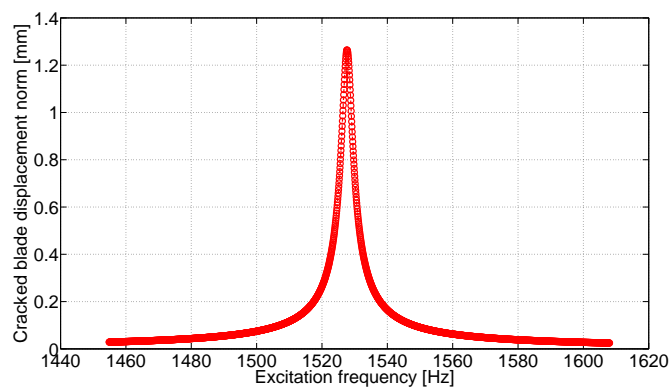

(a)

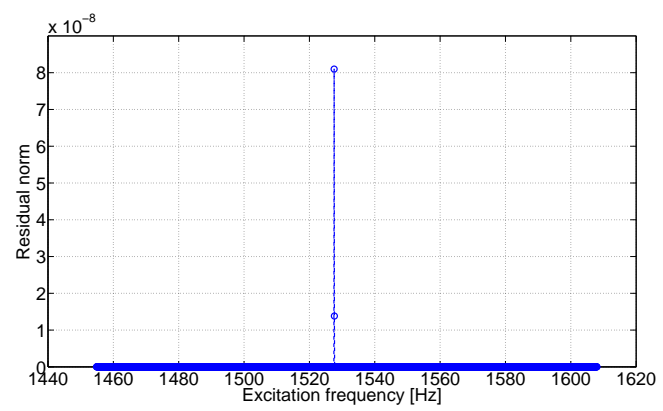

(b)

Figure 6: The (a) amplitude and (b) residual calculated using BAA for the $2^{\text {nd }}$ mode family

of time). The linear momentum can be projected along 3 orthogonal directions in the physical space (given by three vectors $\left.\mathbf{e}_{1}, \mathbf{e}_{2}, \mathbf{e}_{3}\right)$ to obtain $\mathbf{e}_{j}^{\mathrm{T}} \mathbf{L}_{s}(0)=\mathbf{e}_{j}^{\mathrm{T}} \mathbf{L}_{o}\left(T_{o}+T_{s}\right)$ for $j=1,2,3$. Note that vectors $\mathbf{e}_{j}$ depend on the choice of reference coordinate system used in the FE model. If, for example, the choice is to use a global Cartesian reference coordinate system (with unit vectors $\mathbf{i}, \mathbf{j}, \mathbf{k}$ ), then the vector

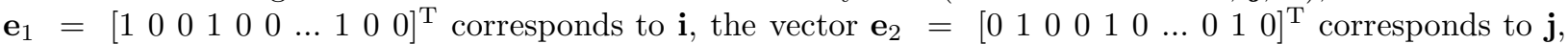
and the vector $\mathbf{e}_{3}=\left[\begin{array}{lllllllll}0 & 0 & 1 & 0 & 0 & 1 & \ldots & 0 & 0\end{array}\right]^{\mathrm{T}}$ corresponds to $\mathbf{k}$. Expressing the linear momentum in terms of $\dot{\mathbf{x}}_{o}$ and $\dot{\mathbf{x}}_{s}$, and using $\dot{\mathbf{x}}_{o} \cong \boldsymbol{\Phi}_{o}^{*} \dot{q}_{o}$ and $\dot{\mathbf{x}}_{s} \cong \boldsymbol{\Phi}_{s}^{*} \dot{q}_{s}$, one obtains $\mathbf{e}_{j}^{\mathrm{T}} \mathbf{M}_{s} \boldsymbol{\Phi}_{s}^{*} \dot{q}_{s}(0)=\mathbf{e}_{j}^{\mathrm{T}} \mathbf{M}_{o} \boldsymbol{\Phi}_{o}^{*} \dot{q}_{o}\left(T_{o}+T_{s}\right)$ for $j=1,2,3$. Next, one can denote $c_{o, j}=\mathbf{e}_{j}^{\mathrm{T}} \mathbf{M}_{o} \boldsymbol{\Phi}_{o}^{*}$ and $c_{s, j}=\mathbf{e}_{j}^{\mathrm{T}} \mathbf{M}_{s} \boldsymbol{\Phi}_{s}^{*}$.

Substituting the energy and momentum expressions into Eq. (6) one obtains

$$
\begin{aligned}
\frac{1}{2} \omega_{s}^{2} q_{s}^{2}\left(T_{s}\right)+\frac{1}{2} \dot{q}_{s}^{2}\left(T_{s}\right)+f_{o} q_{o}\left(T_{s}\right)-f_{s} q_{s}\left(T_{s}\right) & =\frac{1}{2} \omega_{o}^{2} q_{o}^{2}\left(T_{s}\right)+\frac{1}{2} \dot{q}_{o}^{2}\left(T_{s}\right), \\
\left(1-c_{e}\right)\left[\frac{1}{2} \omega_{o}^{2} q_{o}^{2}\left(T_{o}+T_{s}\right)+\frac{1}{2} \dot{q}_{o}^{2}\left(T_{o}+T_{s}\right)\right]+f_{s} q_{s}(0)-f_{o} q_{o}\left(T_{o}+T_{s}\right) & =\frac{1}{2} \omega_{s}^{2} q_{s}^{2}(0)+\frac{1}{2} \dot{q}_{s}^{2}(0), \\
c_{s, j} \dot{q}_{s}\left(T_{s}\right) & =c_{o, j} \dot{q}_{o}\left(T_{s}\right), \\
c_{o, j} \dot{q}_{o}\left(T_{o}+T_{s}\right) & =c_{s, j} \dot{q}_{s}(0),
\end{aligned}
$$

where $j=1,2,3$. There are 8 transition conditions in Eq. (11) for the 5 unknowns, namely $o_{1}, o_{2}, s_{1}, s_{2}$, and $\alpha$. The transition conditions have a nonlinear dependence on the unknowns, therefore various nonlinear solvers can be employed to find the solution. In this work, the function "Isqnonlin" from Matlab was used to solve for the unknowns by minimizing the residual in the 8 relations in Eq. (11).

Using Eq. (4), one can construct $q_{o}$ and $q_{s}$ with the obtained $o_{1}, o_{2}, s_{1}, s_{2}$, and $\alpha$. Then, physical displacement $\mathbf{x}_{o}$ and $\mathbf{x}_{s}$ are calculated using the modal to physical transformation such as $\mathbf{x}_{o} \cong \boldsymbol{\Phi}_{o}^{*} q_{o}$ and $\mathbf{x}_{s} \cong \boldsymbol{\Phi}_{s}^{*} q_{s}$. One steady-state vibration cycle can be constructed using the obtained $\mathbf{x}_{o}$ and $\mathbf{x}_{s}$ with time fraction $f$ as shown in Fig. 1. Finally one can calculate the amplitude of the constructed steady-state 


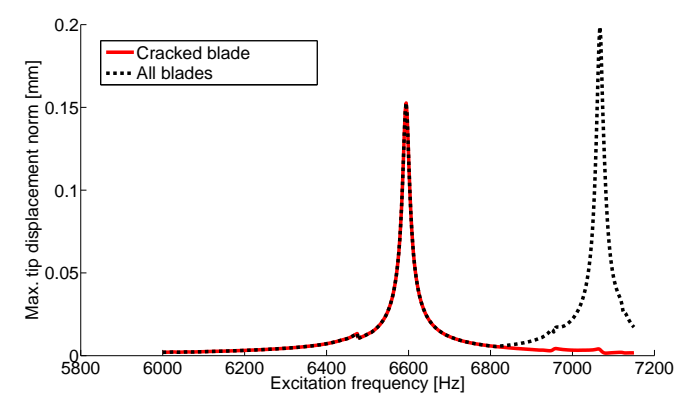

Figure 7: Nonlinear forced response of the $7^{\text {th }}$ mode family

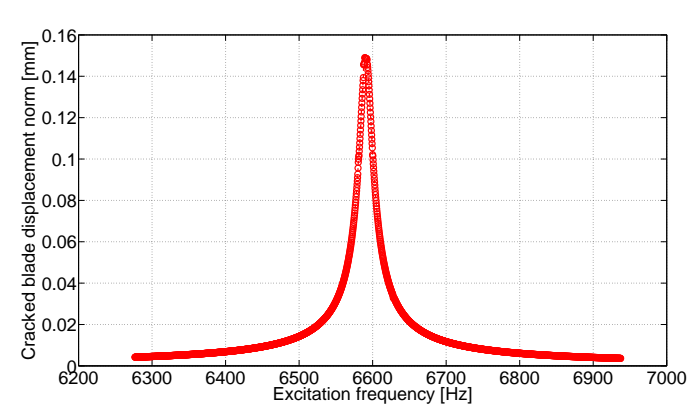

(a)

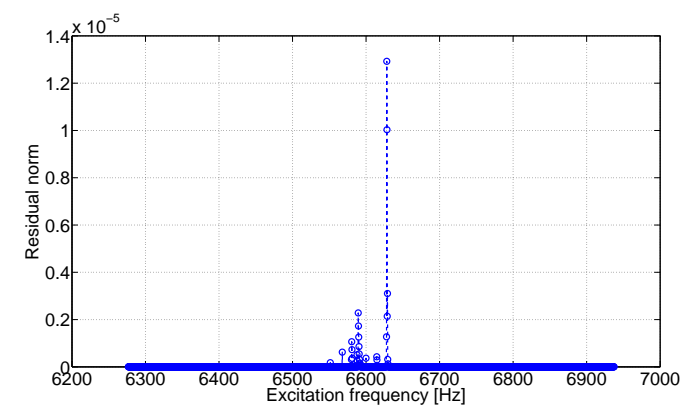

(b)

Figure 8: The (a) amplitude and (b) residual calculated using BAA for the $7^{\text {th }}$ mode family

vibration cycle, which is the approximate amplitude of steady-state vibration of a system with piecewiselinear nonlinearity.

\section{Results}

In this section, the vibration amplitude of a full bladed disk system with piecewise-linear nonlinearity is first calculated using in-house code based on a hybrid frequency/time (HFT) domain solver [25-28]. Engine order excitation 0 with force amplitude of $1 \mathrm{kN}$ was applied at specified frequencies to obtain forced responses. Then, BAA is employed to calculate an approximate amplitude, and the results are compared.

The model used in this work is the tuned bladed disk shown in Fig. 2. The blisk has 20 blades one of which has a crack. The crack length is $37.5 \%$ of the chord on the leading edge at $50 \%$ span from the root of the blade. The material of the bladed disk is a Titanium alloy with Young's modulus $E=114 \mathrm{GPa}$, density $\rho=4,420 \mathrm{~kg} / \mathrm{m}^{3}$, and Poisson's ratio $\nu=0.31$. The full order model has 31,878 degrees of freedom. The damping is modeled as Rayleigh damping (without mass matrix contribution), $\mathbf{C}=\beta \mathbf{K}$, where $\beta$ is a scalar (with a constant value in each frequency range of interest: $\beta=8.37658 \times 10^{-7}$ for the $1^{\text {st }}$ mode family, $\beta=2.09277 \times 10^{-7}$ for the $2^{\text {nd }}$ mode family, $\beta=4.85406 \times 10^{-8}$ for the $7^{\text {th }}$ mode family, and $\beta=2.89031 \times 10^{-8}$ for the $10^{\text {th }}$ mode family). These values correspond to a viscous damping ratio value of $\zeta=0.001$. The commercial software ANSYS was used to obtain the mass and stiffness matrices.

The nonlinear forced response for the $1^{\text {st }}$ mode family was calculated using a HFT method [25] and the results are shown in Fig. 3. Plotted is the tip displacement amplitude of the excited node for the cracked blade and the maximum tip displacement amplitude of all excited nodes (one excited node per blade). The resonant frequency of the cracked blade is $384.3 \mathrm{~Hz}$ and the amplitude at that frequency is $8.105 \mathrm{~mm}$. The bilinear frequency for this mode family was calculated as $\omega_{B F A}=384.94 \mathrm{~Hz}$ using bilinear frequency approximation. This frequency was used for BAA in Eq. (11). The energy loss coefficient $c_{e}$ for this mode family is 0.015. The amplitude and the residual were calculated and are shown in Fig. 4. At the bilinear 


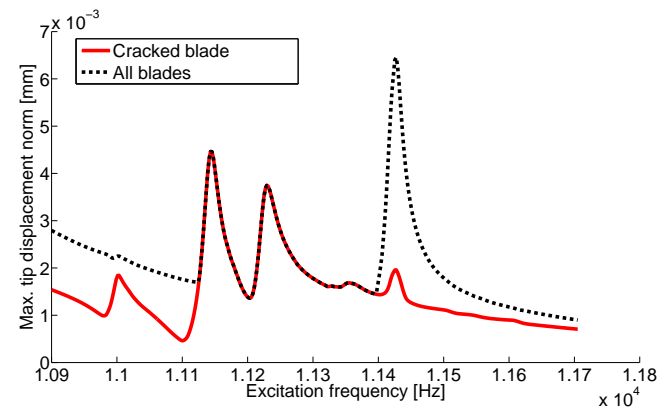

Figure 9: Nonlinear forced response of the $10^{\text {th }}$ mode family

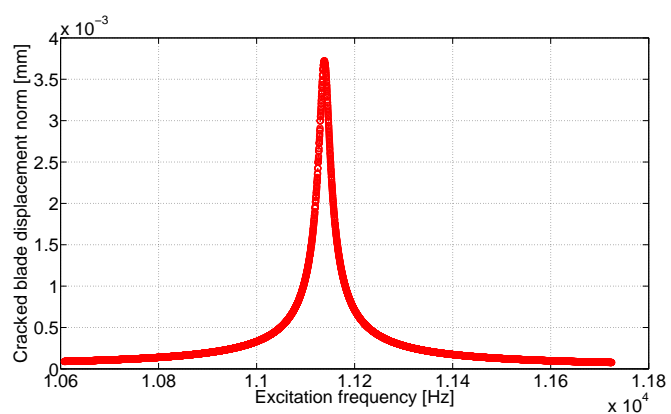

(a)

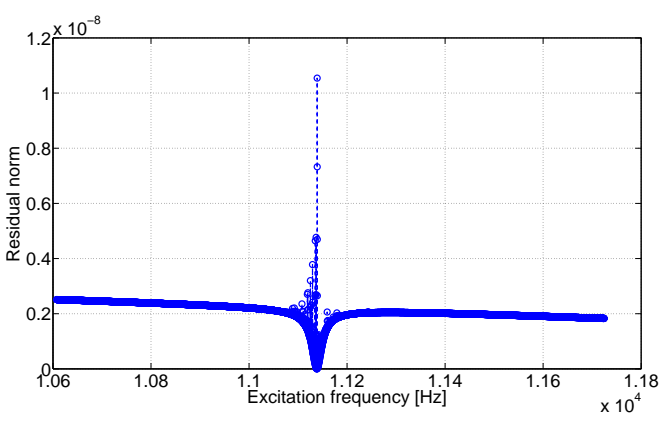

(b)

Figure 10: The (a) amplitude and (b) residual calculated using BAA for the $10^{\text {th }}$ mode family

frequency, the approximated amplitude is $8.337 \mathrm{~mm}$. The relative error of this result compared to that of the nonlinear forced response is $2.86 \%$. Note that the residual is of order $10^{-9}$, which means that all relations in Eq. (11) are satisfied.

For the $2^{\text {nd }}$ mode family, the nonlinear forced response is shown in Fig. 5. The resonant frequency of the cracked blade is $1525.1 \mathrm{~Hz}$ and the amplitude at that frequency is $1.261 \mathrm{~mm}$. The bilinear frequency for this mode family was calculated as $\omega_{B F A}=1527.69 \mathrm{~Hz}$. The coefficient $c_{e}$ for this mode family is 0.0015 . The amplitude and the residual were calculated and are shown in Fig. 6. At the bilinear frequency, the approximated amplitude is $1.264 \mathrm{~mm}$. The relative error of this result compared to that of the nonlinear forced response is $0.24 \%$. Note that the residual is of order $10^{-8}$.

For the $7^{\text {th }}$ mode family, the nonlinear forced response is shown in Fig. 7 . The resonant frequency of the cracked blade is $6594.14 \mathrm{~Hz}$ and the amplitude at that frequency is $0.1527 \mathrm{~mm}$. The bilinear frequency for this mode family was calculated as $\omega_{B F A}=6590.85 \mathrm{~Hz}$. The coefficient $c_{e}$ for this mode family is 0.004 . The amplitude and the residual were calculated and are shown in Fig. 8. At the bilinear frequency, the approximated amplitude is $0.1487 \mathrm{~mm}$. The relative error of this result compared to that of the nonlinear forced response is $2.62 \%$. Note that the residual is of order $10^{-5}$.

For the $10^{\text {th }}$ mode family, the nonlinear forced response is shown in Fig. 9. The resonant frequency of the cracked blade is $11,144.34 \mathrm{~Hz}$ and the amplitude at that frequency is $0.004294 \mathrm{~mm}$. The bilinear frequency for this mode family was calculated as $\omega_{B F A}=11,138.37 \mathrm{~Hz}$. The coefficient $c_{e}$ for this mode family is zero. The amplitude and the residual were calculated and are shown in Fig. 10. At the bilinear frequency, the approximated amplitude is $0.003722 \mathrm{~mm}$. The relative error of this result compared to that of the nonlinear forced response is $13.3 \%$. Note that the residual is of order $10^{-8}$. 


\section{Conclusions}

An efficient and novel methodology to approximate the steady-state amplitude of vibration of a system with piecewise-linear nonlinearity was developed. The method breaks each cycle of the steady-state nonlinear response into two parts. The first portion of the response corresponds to the open case (if there is a crack that would mean that the crack is open). The second portion of the response corresponds to the sliding case (if there is a crack that would mean the crack is closed). The transition between each state is assumed to take place quickly. The method then enforces transitional compatibility at the contacting surfaces, namely that energy and momentum are conserved when the system transitions from the open to sliding and sliding to open cases. The method was demonstrated on full blisk model with a crack and found to obtain very accurate results compared to a nonlinear analysis.

\section{References}

${ }^{1}$ Parkinson, J. S. and Wynne, R. J., "Reduced-Order Modeling and State Estimation applied to gas distribution systems," IEEE Transactions on Automatic Control, Vol. AC-31, No. 8, 1986, pp. 701-708.

${ }^{2}$ Ramakrishnan, J. V., Rao, S. V., and Koval, L. R., "Reduced-Order Modeling of Flexible Structures," J. Guidance, Vol. 11, No. 5, 1988, pp. 459-464.

${ }^{3}$ Tsuchiya, K., Kashiwase, T., and Yamada, K., "Reduced-Order Models of a Large Flexible Spacecraft," J. Guidance, Vol. 12, No. 6, 1989

${ }^{4}$ Yang, B. and Chen, H., "Redued-order shaft system models of turbogenerators," IEEE Transactions on Power Systems, Vol. 8, No. 3, 1993, pp. 1366-1374.

${ }^{5}$ Castanier, M. P., Ottarsson, G., and Pierre, C., "A Reduced Order Modeling Technique for Mistuned Bladed Disks," Journal of Vibration and Acoustics, Vol. 119, 1997, pp. 439-447.

${ }^{6}$ Tang, D., Conner, M. D., and Dowell, E. H., "Reduced-Order Aerodynamic Model and Its Application to a Nonlinear Aeroelastic System," Journal of aircraft, Vol. 35, No. 2, 1998, pp. 332-338.

${ }^{7}$ Mohiuddin, M. A., Bettayeb, M., and Khulief, Y. A., "Dynamic analysis and reduced order modelling of flexible rotorbearing systems," Computers and Structures, Vol. 69, 1998, pp. 349-359.

${ }^{8}$ Bladh, R., Castanier, M. P., and Pierre, C., "Reduced Order Modeling and Vibration Analysis of Mistuned Bladed Disk Assemblies With Shrouds," Journal of Engineering for Gas Turbines and Power, Vol. 121, 1999, pp. 515-522.

${ }^{9}$ Friswell, M. I. and Inman, D. J., "Reduced-Order Models of Structures with Viscoelastic Components," AIAA Journal, Vol. 37, No. 10, 1999, pp. 1318-1325.

${ }^{10} \mathrm{He}$, Z., Epureanu, B. I., and Pierre, C., "Fluid-Structural Coupling Effects on the Dynamics of Mistuned Bladed Disks," AIAA Journal, Vol. 45, No. 3, 2007, pp. 552-561.

${ }^{11} \mathrm{He}$, Z., Epureanu, B. I., and Pierre, C., "Parametric Study of the Aeroelastic Response of Mistuned Bladed Disks," Computers and Structures, Vol. 85, No. 11-14, 2007, pp. 852-865.

${ }^{12} \mathrm{He}, \mathrm{Z}$., Epureanu, B. I., and Pierre, C., "Convergence Predictions for Aeroelastic Calculations of Tuned and Mistuned Tuned Bladed Disks," Journal of Fluids and Structures, Vol. 24, No. 5, 2008, pp. 732-749.

${ }^{13}$ Madden, A., Castanier, M. P., and Epureanu, B. I., "Reduced-Order Model Construction Procedure for Robust Mistuning Identification of Blisks," AIAA Journal, Vol. 46, No. 11, 2008, pp. 2890-2898.

${ }^{14}$ D'Souza, K. and Epureanu, B. I., "A Statistical Characterization of the Effects of Mistuning in Multistage Bladed Disks," Journal of Engineering for Gas Turbines and Power, Vol. 134, No. 1, 2012, pp. 012503.

${ }^{15} \mathrm{Li}$, D. F. and Gunter, E. J., "Compenet mode synthesis of large rotor systems," Journal of engineering for powertransactions of the ASME, Vol. 104, 1982, pp. 552-560.

${ }^{16}$ Moon, J. and Cho, D., "A component mode synthesis applied to mechanisms for an investigation of vibration," Journal of sound and vibration, Vol. 157, 1992, pp. 67-79.

${ }^{17}$ Bampton, M. C. C. and Craig, R. R., "Coupling of Substructures for Dynamic Analyses," AIAA Journal, Vol. 6, No. 7, 1968, pp. 1313-1319.

${ }^{18}$ Friswell, M. I., Penny, J. E. T., and Garvey, S. D., "Using linear model reduction to investigate the dynamics of structures with local non-linearities," Mechanical Systems and Signal Processing, Vol. 9, 1995, pp. 317-328.

${ }^{19}$ Friswell, M. I., Penny, J. E. T., and Garvey, S. D., "The application of the IRS and balanced realization methods to obtain reduced models of structures with local non-linearity," Journal of Sound and Vibration, Vol. 196, 1996, pp. 453-468.

${ }^{20}$ Segalman, D. J., "Model reduction of systems with localized nonlinearities," Journal of Computational and Non-linear Dynamics, Vol. 2, 2007, pp. 249-266.

${ }^{21}$ Chen, S. L. and Shaw, S. W., "Normal modes for piecewise linear vibratory systems," Nonlinear Dynamics, Vol. 10, 1996, pp. 135-164.

${ }^{22}$ Jiang, D., Pierre, C., and Shaw, S. W., "Large-amplitude non-linear normal modes of piecewise linear systems," Journal of Sound and Vibration, Vol. 272, 2004, pp. 869-891.

${ }^{23}$ Apiwattanalunggarn, P., Shaw, S. W., and Pierre, C., "Component mode synthesis using nonlinear normal modes," Nonlinear Dynamics, Vol. 41, 2005, pp. 17-46.

${ }^{24}$ Saito, A. and Epureanu, B. I., "Bilinear Modal Representations for Reduced-Order Modeling of Localized Piecewise-linear Oscillators," Journal of Sound and Vibration, Vol. 330, 2011, pp. 3442-3457.

${ }^{25}$ Saito, A., Castanier, M. P., and Pierre, C., "Effects of a cracked blade on mistuned turbine engine rotor vibration," Journal of Vibration and Acoustics, Vol. 131, No. 6, 2009, pp. 061006. 
${ }^{26}$ Saito, A., Castanier, M. P., Pierre, C., and Poudou, O., "Efficient nonlinear vibration analysis of the forced response of rotating cracked blades," Journal of Computational and Nonlinear Dynamics, Vol. 4, No. 1, 2009, pp. 011005.

${ }^{27}$ Saito, A., Epureanu, B. I., Castanier, M. P., and Pierre, C., "Node Sampling for Nonlinear Vibration Analysis of Structures with Intermittent Contact," AIAA Journal, Vol. 48, No. 9, 2010, pp. 1903-1915.

${ }^{28}$ D'Souza, K., Saito, A., and Epureanu, B. I., "Reduced-Order Modeling for Nonlinear Analysis of Cracked Mistuned Multistage Bladed-Disk Systems," AIAA Journal, Vol. 50, No. 2, 2012, pp. 304-312.

${ }^{29}$ Shaw, "A periodically forced piecewise linear oscillator," Journal of Sound and vibration, Vol. 90, No. 1, 1983, pp. 129155 